REFERENCIA: García, R.A., Llapa, M., Bartesaghi, W., \& Torres, J. (2020). Diseño y validación de un cuestionario de interés en el aprendizaje de las ciencias básicas. ENSAYOS, Revista de la Facultad de Educación de Albacete, 35(1). Enlace web: http://www.revista.uclm.es/index.php/ensayos - Consultada en fecha (dd-mm-aaaa)

\title{
DISEÑO Y VALIDACIÓN DE UN CUESTIONARIO DE INTERÉS EN EL APRENDIZAJE DE LAS CIENCIAS BÁSICAS ${ }^{1}$

\author{
DESIGN AND VALIDATION OF A QUESTIONNAIRE OF INTEREST IN \\ LEARNING BASIC SCIENCES
}

\author{
Raúl Alberto Garcia Castro \\ rgarciac@unjbg.edu.pe \\ Martín Pedro Llapa Medina \\ mllapam@unjbg.edu.pe \\ Willian Bartesaghi Aste \\ wbartesaghia@unjbg.edu.pe \\ Julio Torres Chávez \\ jtorresc@unjbg.edu.pe
}

Departamento de Ciencias Formales y Naturales. Universidad Nacional Jorge Basadre Grohmann (Perú)

Recibido: $21 / 01 / 2020$

Aceptado: 29/05/2020

\begin{abstract}
Resumen:
El objetivo fue diseñar y validar, un cuestionario para medir el interés individual en el aprendizaje de las ciencias básicas. La validación de contenido se realizó con la revisión bibliográfica, la opinión de 6 expertos y una prueba piloto con 50 alumnos, el instrumento inicial contiene 18 ítems. Luego se realizó la validación de constructo, con 672 alumnos de secundaria de 22 colegios, dividida en dos grupos de 336 . El cuestionario se aplicó al $1^{\circ}$ grupo y se realizó el AFE, la prueba permitió identificar 4 factores latentes y 16 ítems. Las dimensiones emergentes son: afecto, intencionalidad, valoración y predisposición. El AFC realizado a los 15 días, confirmo el resultado del AFE, la prueba de fiabilidad proporciono índices $>0,79$. Se comprobó que el instrumento es válido para medir el interés individual, en alumnos de secundaria, entre 11 y 18 años, sus enunciados pueden adecuarse a otras materias.
\end{abstract}

Palabras clave: Interés individual, cuestionario, aprendizaje de las ciencias básicas.

\footnotetext{
${ }^{1}$ Proyecto financiado con fondos del Canon, Sobre canon y Regalías Mineras - UNJBG
} 


\begin{abstract}
:
The objective was to design and validate a questionnaire to measure individual interest in learning basic sciences. Content validation was carried out with the bibliographic review, the opinion of 6 experts and a pilot test with 50 students, the initial instrument contains 18 items. Then the construct validation was carried out, with 672 high school students from 22 schools, divided into two groups of 336 . The questionnaire was applied to the 1 st group and the AFE was performed, the test allowed to identify 4 latent factors and 16 items. The emerging dimensions are: affection, intentionality, assessment and predisposition. The AFC performed at 15 days, I confirm the result of the AFE, the reliability test provided indexes $>0.75$. It was found that the instrument is valid to measure individual interest, in high school students, between 11 and 18 years, their statements can be adapted to other subjects.
\end{abstract}

Keywords: Individual interest, questionnaire, learning of basic sciences.

\title{
1. Introducción
}

El avance de las ciencias depende del desarrollo de las capacidades de las futuras generaciones, darle sostenibilidad al desarrollo económico de un país, en un escenario globalizado, significa darle importancia a la investigación (Hazelkorn et al., 2015). Esto requiere el desarrollo de capacidades que comprometen el dominio de las ciencias básicas. Una rápida mirada sobre la situación, reporta que los alumnos de la educación secundaria están menos interesados en las ciencias. El interés por el aprendizaje de las ciencias básicas en comparación a otras materias está por debajo de sus preferencias. Lo que minimiza las posibilidades de un país, porque sus futuras generaciones tendrán dificultades para emprender proyectos de innovación, mediante la investigación científica. Esta situación se aprecia en diferentes países de Europa (Rocard et al., 2007) y Latinoamérica (Busquets, Silva y Larrosa, 2016).

El aprendizaje de las ciencias básicas, tiene que ver con un proceso interno que sucede en la mente del aprendiz, en la escuela el profesor da la iniciativa y propicia las actividades para que los alumnos aprendan. A esto se suma una connotación personal, que nace como el resultado del esfuerzo encausado por el propio estudiante, al sentir satisfacción ante una necesidad de aprender (Rennier y Punch, 1991; Steinkamp y Maehr, 1983). Dentro de este proceso el interés juega un papel importante debido a que el alumno aprende en la medida que sienta que está interesado en un conocimiento determinado. El interés es un factor eficiente que mueve la actividad mental del alumno, es un sentimiento agradable o desagradable producido por una idea u objeto con el poder de captar y mantener la atención (Kelly, 1982).

El interés es una variable multifacética, es un fuerte componente, combustible de la motivación por que desencadena un proceso en el cual, las personas se sienten motivadas por realizar una tarea, lo mantienen en el tiempo y se vuelven a comprometer con los objetivos de la tarea. Despierta la atención y tiene poder para aumentarla o reducirla, ya que lo nuevo o complejo provoca la necesidad de involucrarnos para comprenderlo a través de la experimentación (Berlyne, 1960; Silvia, 2005). La explicación del porqué las personas se auto regulan para mantener fuertes niveles al realizar una tarea y no sentirse agotados, también fue estudiado por O'Keefe y Linnenbrink (2014), demostrando que el interés desempeña un papel importante en el mantenimiento de la energía mental. La literatura distingue dos tipos: el interés individual (Ainley, 2008) y el situacional (Hidi, 1986), el primero tiene arraigo en las ciencias, música, deportes distracciones, entre otros (Schiefele, 1991), es profundo y se considera como una predisposición para volver a participar en una actividad conservándose a lo largo del tiempo 
(Hidi, 2006). En cambio, el interés situacional es fugaz, por que despierta por las condiciones ambientales que brindan estímulos externos (Rotgans, 2011, 2014)

Cuando tratamos el interés como concepto, nos remitimos a exposición individual en el ámbito de la escuela, específicamente en relación al aprendizaje de las distintas materias del currículo, por otro lado, el termino está muy vinculado con la psicología y las emociones del estudiante, por tanto, es un sentimiento que mueve las esferas de nuestros sentidos y hace que fijemos nuestra atención en algún acontecimiento. Muchos autores han escrito al respecto y conciben al interés individual como una preferencia relativamente duradera a ciertas áreas del conocimiento o actividades (Schiefele, 1991; 1992); Por el lado, educativo se aborda el concepto en función del conocimiento y la valoración que realizan los alumnos (sobre una materia o curso), que tiene la capacidad de despertar el deseo de implicarse en actividades relacionadas con el aprendizaje de ese campo del conocimiento (Boekaerts y Boscoso, 2002).

La evaluación del interés por el aprendizaje de las ciencias, motivo el trabajo de muchos investigadores, sus conclusiones concuerdan que el interés se caracteriza esencialmente por su carácter interno (Krapp, 1992), es una variable relacionada con las emociones del aprendiz y el valor que le otorgan a los hechos académicos. Ambos componentes se combinan para darle significado personal a los contenidos del aprendizaje. El desarrollo de los enfoques de la motivación intrínseca, abrieron el camino para el desarrollo de la investigación del interés (Krapp, 1992; Ren-Ninger, Hidi y Krapp, 1992). Un concepto integrado por tres componentes, cognitivo, emocional y el valor, dio lugar a la construcción de un instrumento para medir el interés; una primera versión del cuestionario (FSI) fue desarrollada por Winteler y Sierwald (1987) y Winteler et al. (1988), incluye el componente de interés emocional relacionado con el valor. Una versión modificada denominado cuestionario SIQ fue publicado por Schiefele, Krapp, Wild y Schrever, I. (1993), el instrumento se puede aplicar a estudiantes por materia de la escuela secundaria o universidad. Por otro lado, el cuestionario Q21, fue utilizado en la prueba PISA 2006, para medir el interés general de los estudiantes por las ciencias divididos por dos subcategorías cognitivas y afectivas. se compone de una escala unidimensional. En el 2015, Rotgans publico la validación del cuestionario de interés individual (QII), el cuestionario tiene tres componentes: cognitivo, comportamiento y actitudes. A pesar de las investigaciones realizadas, el interés individual mantiene una condición compleja en su constructo, de ahí que, aun requiere el desarrollo de modelos teóricos que posibiliten la construcción de instrumentos válidos y confiables para medirlo en los diferentes contextos educativos (Rotans, 2015).

En la presente investigación se ha tomado como base el modelo integrado por el componente: emocional, valor y la intencionalidad (Schiefele, 1991, 1999 y Krapp y Lewalter, 2001), ya que es un modelo que está presente con una, dos dimensiones en los diferentes instrumentos publicados. El objetivo de esta investigación es contribuir a mejorar el conocimiento sobre el interés, mediante el diseño y la validación de un instrumento que permita medir el interés personal por el aprendizaje de las ciencias básicas, en alumnos de la educación secundaria, validando las dimensiones y los ítems correspondientes.

\section{Método}

\subsection{Procedimiento}

Considerando que aún no existe un consenso claro sobre la conceptualización del interés individual y su medición (Renninger, y Hidi, 2011; Rotgans, 2015), el diseño y validación del instrumento, requirió en primer lugar de la validación de contenido (Mitchell, 1986, citado en Ding y Hershberger, 2002). El procedimiento consistió en una revisión bibliográfica, respecto a los fundamentos teóricos y las publicaciones de los instrumentos de diferentes autores; obteniendo como producto un listado de enunciados que luego fue sometido a consulta de 
expertos en la materia. Seguidamente el borrador se sometió a una prueba piloto de 50 alumnos de educación secundaria, que fueron elegidas al azar, pertenecientes a 4 instituciones educativas. Los resultados permitieron realizar las correcciones y modificaciones correspondientes, adecuándolas a las características de la población. En segundo lugar, el cuestionario corregido se sometió a validez de constructo (Pérez, et al, 2000) mediante el análisis factorial exploratorio (AFE), el procedimiento consistió, en un análisis multivariado, que tiene como finalidad identificar la estructura de factores subyacentes a partir de un conjunto amplio de datos (Blalock 1966). Esto posibilito otorgar significado teórico al conjunto de factores que componen el modelo a partir de los ítems del cuestionario (Martínez Arias 1995). El AFE se estimó sobre la matriz de correlaciones policóricas, adecua-do para variables categóricas con el programa FACTOR versión 10.8 (Lorenzo-Seva y Ferrando 2006); se utilizó el método de extracción Minimum Rank Factor Analysis (Ten Berge y Kiers 1991), con rotación oblicua Promax (Dien 2010; Hendrickson y White 1964; Tabachnick y Fidell 2007). Se tomó como criterio de correspondencia de ítems, una carga factorial por encima de 0.3 (Hogarty, et al., 2005), también que el número de ítems por factor sea mayor a 3 (Velicer y Fava 1998); El coeficiente (KMO) de Kaiser-Mayer-Olikin (Kaiser 1970; Thompson 2008) se aplicó para verificar la correlación entre los ítems (magnitudes que fluctúan entre 0 y1); la prueba de esfericidad de Bartlett permitió verificar si la matriz de datos es apropiada para realizar un análisis factorial exploratorio (Bartlett, 1950, 1951; Gorsuch 1973; Yanai y Ichikawa, 2007); La evaluación de la bondad de ajuste del modelo factorial se realizó mediante el cuadrado medio de la raíz de residuos (RMSR) y el valor medio esperado de RMSR para un modelo aceptable (criterio de Kelley 1935, página 146; véase también Harman, 1962, página 21 de la 2ª edición).

El cuestionario estructurado con sus respectivas dimensiones identificadas en el AFE, a los 15 días nuevamente se aplicó el cuestionario y con esta información se realizó el análisis Factorial confirmatorio (AFC). Este procedimiento posibilito una mayor contrastación de las hipótesis especificadas (Bollen 1989). El análisis del ajuste del modelo se realizó utilizando el software para análisis de datos categóricos Mplus (Muthén 2004). Los estimadores aplicados se basaron en correlaciones tetracónicas para variables categóricas (Muthén, 1998 y Muthen, 2004). Seguidamente el instrumento se sometió a un análisis de confiabilidad para verificar su consistencia interna de cada uno de los factores, este procedimiento se realizó con el programa estadístico R estudio para datos ordinales.

\subsection{Participantes}

Para la etapa de validez del contenido de contenido se seleccionó una muestra piloto de 50 alumnos a criterio del investigador y con selección al azar simple. También se recurrió a la opinión de 6 expertos en la materia. Para el proceso de la validación de constructo se recurrió a la información proveniente de fuentes primarias, conformada por alumnos de 22 instituciones educativas públicas y privadas de la Región de Tacna-Perú. La muestra estuvo conformada por 672 alumnos divididos en dos grupos, 336 para la prueba AFC y 336 para la prueba de AFC. La selección de las unidades muestrales del AFE fue al azar, conformada por alumnos del 1ㅇ, 2으, 3을, 4 ㅇ y 5 o año de educación secundaria, de ambos sexos, donde el $53.5 \%$ fueron mujeres y el $46.5 \%$ varones, cuyas edades fluctúan entre 11 y 18 años, siendo el promedio de edad cronológica de 15,1 años. El análisis factorial confirmatorio (AFC), se realizó con una muestra de 336 alumnos, el procedimiento de selección muestral fue similar al AFE. El 53.4\% fueron mujeres y el $46.6 \%$ varones, las edades fluctúan entre 11 y 18 años, con un promedio de edad de 15,6 años. 


\subsection{Diseño del cuestionario}

El diseño se realizó a partir de la revisión bibliográfica profunda de los artículos relacionados a la evaluación del interés individual. Mediante este procedimiento se pudieron identificar patrones comunes en la previsión de los diferentes cuestionarios, adicionalmente se trabajó con los componentes dimensionales propuestos por: Schiefele $(1991 ; 1999)$ y Krapp y Lewalter (2001). La suma de estos procedimientos, dieron lugar a una matriz compuesta por un listado de enunciados. Seguidamente la matriz se sometió a validación por la opinión de expertos, con el objeto de verificar la concordancia de los enunciados; como resultado de este proceso se modificaron varios enunciados y se incluyeron otros. Sobre la base de la matriz seleccionada en la etapa anterior se procedió a la construcción del cuestionario con escala tipo Likert (De Vellis, 2012 y Furr 2011). La primera versión del cuestionario se aplicó a una muestra piloto de 50 alumnos para detectar posibles problemas en su aplicación, comprobar la comprensión de las preguntas y evaluar la pertinencia de la redacción de los ítems. El cuestionario corregido en su versión inicial consta de 18 ítems, con tres dimensiones: componente emocional, componente de valor y el componente de intencionalidad. Para cada ítem, se formularon enunciados que motivan la respuesta del alumno, con escala de respuestas de 4 categorías: totalmente de acuerdo, de acuerdo, en desacuerdo, y totalmente de acuerdo. Los enunciados de los ítems se formularon en razón del contexto local, donde las ciencias básicas están representadas por: las matemáticas y CTA (ciencia tecnología y ambiente) dentro de CTA se concentran: biología, química y física. Sin embargo, los enunciados se pueden redactar optando por cualquier materia especifica (matemáticas, biología, química y/o física). El cuestionario está diseñado para ser aplicado en adolescentes de educación secundaria, entre 11-18 años.

\section{Resultados}

En este apartado se presentan los resultados de los AFE, AFC, análisis de fiabilidad y los descriptivos.

\subsection{Resultados del análisis factorial exploratorio (AFE)}

La información obtenida en la primera aplicación del cuestionario se procesó mediante programa FACTOR versión 10.8. Se estimó la correlación entre los ítems, proporcionándonos un valor de Kaiser-Meyer- Olkin (KMO) de 0.872, que representa un nivel alto (Beavers et al. 2013). La prueba de esfericidad de Bartlett's $=1295,5(\mathrm{df}=153 ; p=0,00001) \mathrm{p}<0,001$, confirma que la prueba es significativa para realizar un AFE. La prueba de extracción de los factores el programa sugirió una solución de 5 factores subyacentes, también se realizaron pruebas de descarte probando con soluciones de 2, 3, 4 y 6 factores, las soluciones no satisfacían el modelo teórico de interés individual. Se descartaron estas soluciones quedándonos con la solución sugerida por el programa de 5 factores subyacentes. La solución explica un $83,34 \%$ de la varianza total, las dimensiones agrupan a los ítems y quedan organizadas según la tabla 1.

En la tabla 1 se aprecia que las cargas factoriales de la comunalidades de los ítems son >a 0.3, están por encima del valor absoluto sugerido, no se recomienda eliminar ítems, apoyados en este criterio. Sin embargo, el factor F4 solo contiene dos factores, por tanto, eliminamos este factor (ítems 15 e ítems 18) criterio recomendado por: Velicer y Fava, 1998, en consecuencia, el instrumento queda con 4 factores que agrupan 16 ítems. Además, las pruebas de descarte estimaron soluciones obteniendo un $\mathrm{KMO}=0.872$ para todos los casos, pero con varianzas explicadas menores a $0.78 \%$. En consecuencia, se descartaron estas soluciones quedándonos con la solución sugerida por el programa. 
Tabla 1. Coeficientes del Análisis Exploratorio

\begin{tabular}{|c|c|c|c|c|c|}
\hline Ítem & F1 & $\mathrm{F} 2$ & F3 & F4 & F5 \\
\hline a1 & & 0.754 & & & \\
\hline a2 & 0.673 & & & & \\
\hline a3 & & 0.572 & & & \\
\hline a4 & 0.520 & & & & \\
\hline a5 & & 0.524 & & & \\
\hline a6 & & 0.756 & & & \\
\hline a7 & 0.687 & & & & \\
\hline a8 & & & & & 0.441 \\
\hline a9 & & & & & 0.551 \\
\hline a10 & & & & & 0.577 \\
\hline a11 & & & & & 0.680 \\
\hline a12 & & & & & 0.673 \\
\hline a13 & & 0.464 & & & \\
\hline a14 & & & 0.485 & & \\
\hline a15 & & & & 0.358 & \\
\hline a16 & & & 0.738 & & \\
\hline a17 & & & 0.662 & & \\
\hline a18 & & & & 0.461 & \\
\hline
\end{tabular}

\subsection{Resultados del análisis factorial confirmatorio (AFC)}

Con el objeto de corregir o corroborar, el modelo identificado en el AFE, se procedio nuevamente aplicar el cuestionario con 16 ítems, con la información recogida se realizó el AFC con Mplus (Muthén 2004). La prueba posibilita una mayor contrastación de las hipótesis especificadas (Bollen 1989). El análisis del ajuste del modelo se presenta en la Tabla 2. Estos resultados confirman que el modelo presenta un ajuste aceptable a los datos: el índice de ajuste comparativo $\mathrm{CFI}=0,901$, está dentro del nivel de los parámetros deseados y el $\mathrm{TLI}=0.892$, ligeramente por debajo de $>0.09$, el error cuadrado medio de aproximación RMSEA $=0,064$, se considera un modelo plausible (Kaplan, 2009; Mulaik 2009).

Tabla 2. Resultados estadísticos de ajuste robustos para los modelos de AFC

\begin{tabular}{llllllll}
\hline Modelo & $\chi 2$ & g.l. & p-valor & CFI & TLI & RMSEA & SRMR \\
\hline 1 & 261,942 & 132 & 0,00 & 0,901 & 0,892 & 0,064 & 0,058 \\
\hline
\end{tabular}

Los índices de estimación indican que los factores del modelo tienen cargas mayores $>0.3$ (Tabla 3) por lo que, se juzga correcto mantener en el modelo con los 16 ítems. 
Tabla 3. Cargas factoriales de los ítems

\begin{tabular}{|c|c|c|c|}
\hline Dimensión & Ítems & $\begin{array}{l}\text { Cargas } \\
\text { factorial }\end{array}$ & Enunciado del ítems \\
\hline \multirow[t]{3}{*}{$\begin{array}{l}\text { Dimensión 1: } \\
\text { Predisposición }\end{array}$} & 2 & 0,673 & $\begin{array}{l}\text { No me gusta hablar mucho sobre los temas relacionados con } \\
\text { las matemáticas o CTA. Prefiero hablar de mis pasatiempos. }\end{array}$ \\
\hline & 4 & 0,52 & $\begin{array}{l}\text { Hacer las tareas de matemática o CTA, me pone de mal } \\
\text { humor }\end{array}$ \\
\hline & 7 & 0,687 & $\begin{array}{l}\text { Para ser absolutamente honesto/a, a veces me siento } \\
\text { bastante indiferente hacia las áreas de matemática o CTA } \\
\text { (Química, Biología-fisca). }\end{array}$ \\
\hline Dimensión 2: & 1 & 0,754 & Estudiar las matemáticas o CTA, es mi actividad favorita. \\
\hline \multirow[t]{4}{*}{ afecto } & 3 & 0,572 & $\begin{array}{l}\text { Me siento capaz de aprender las matemática o CTA, sin } \\
\text { ayuda de los demás }\end{array}$ \\
\hline & 5 & 0,524 & $\begin{array}{l}\text { Cuando estoy en una biblioteca o librería, me gusta revisar } \\
\text { las revistas o libros que tienen que ver con temas } \\
\text { relacionados con las matemática o CTA. }\end{array}$ \\
\hline & 6 & 0,756 & Disfruto mucho durante las clases de matemáticas o CTA \\
\hline & 13 & 0,464 & $\begin{array}{l}\text { Aunque no se requieran para pasar el ano, estudiaría } \\
\text { intensamente las áreas de matemática y CTA }\end{array}$ \\
\hline \multirow{3}{*}{$\begin{array}{l}\text { Dimensión 3: } \\
\text { Intencionalidad }\end{array}$} & 14 & 0,485 & $\begin{array}{l}\text { Las matemáticas, la química, la biología o la física (CTA), } \\
\text { están muy relacionadas con mis objetivos en el futuro. }\end{array}$ \\
\hline & 16 & 0,738 & $\begin{array}{l}\text { Creo que trabajar en el futuro en una carrera científica } \\
\text { (laboratorios) resulta ser muy aburrido para mí. }\end{array}$ \\
\hline & 17 & 0,662 & $\begin{array}{l}\text { Estoy muy interesado en los fenómenos o tecnología, que se } \\
\text { puedan explicar científicamente }\end{array}$ \\
\hline \multirow{5}{*}{$\begin{array}{l}\text { Dimensión 4: } \\
\text { Valoracion }\end{array}$} & 8 & 0,441 & $\begin{array}{l}\text { Siento que los temas de matemática o CTA, contribuyen } \\
\text { mucho a mi desarrollo personal }\end{array}$ \\
\hline & 9 & 0,551 & $\begin{array}{l}\text { Me interesa las áreas de matemática y CTA, porque, elevan } \\
\text { mi autoestima personal. }\end{array}$ \\
\hline & 10 & 0,577 & $\begin{array}{l}\text { En la vida real, los conocimientos que recibo de las áreas de } \\
\text { matemáticas y CTA, están contribuyendo para que yo sea } \\
\text { más competente ante los demás. }\end{array}$ \\
\hline & 11 & 0,680 & $\begin{array}{l}\text { Siento que los conocimientos sobre las matemáticas y CTA, } \\
\text { me ayudan a resolver problemas cotidianos }\end{array}$ \\
\hline & 12 & 0,673 & $\begin{array}{l}\text { La mayoría de los conocimientos sobre matemática y CTA, } \\
\text { que desarrolla el profesor (a) están muy relacionados con } \\
\text { mis experiencias personales. }\end{array}$ \\
\hline
\end{tabular}

En la tabla 4 se observa que las cargas factoriales entre las dimensiones observadas presentan valores $>0.3$, lo cual, corrobora que el modelo con los 4 factores es aceptable. 
Tabla 4. Cargas factoriales entre las dimensiones

\begin{tabular}{|l|l|l|l|l|l|}
\hline D1 & D1 & D1 & D2 & D2 & D3 \\
\hline D2 & D3 & D4 & D3 & D4 & D4 \\
\hline 0.558 & 0.626 & 0.55 & 0,49 & 0.645 & 0.63 \\
\hline
\end{tabular}

\subsection{Resultados descriptivos y fiabilidad}

El análisis de confiabilidad posibilito evaluar la consistencia interna de cada uno de los factores. El análisis se realizó con el programa estadístico $R$ estudio para datos ordinales, los resultados se presentan en la tabla 5 , donde se observa que las dimensiones alcanzaron coeficientes altos, que fluctúan entre 0.79 (valoración) y 0.92 (intencionalidad). El nivel de fiabilidad de las dimensiones, superan la magnitud de 0.75 . Los resultados de los intervalos de confianza al $95 \%$, indican que el nivel de fiabilidad aceptable.

Tabla 5. Coeficientes

\begin{tabular}{lllll}
\hline № & Dimensión & № ítems & Media & Alfa ordinal \\
\hline 1 & Predisposición & 3 & 2,69 & 0.82 \\
2 & Afecto & 5 & 2,7 & 0.89 \\
3 & Intencionalidad & 3 & 3,2 & 0,92 \\
4 & Valoracion & 5 & 2,72 & 0.79 \\
\hline
\end{tabular}

\section{Discusiones}

El diseño del instrumento requirió la construcción de una matriz condicionada compuesta de enunciados elaborados sobre la base de la revisión de diversos modelos teóricos y cuestionarios y la opinión de expertos. Este trabajo de validación de contenido permitió capturar más elementos para la construcción del concepto de interés individual. Los resultados con la prueba de análisis factorial exploratorio (AFE), mediante el programa FACTOR versión 10.8 (LorenzoSeva y Ferrando 2006), sugirió una solución con 5 factores subyacentes que explica un 83,34\% de la varianza total, con cargas factoriales $>0,3$, a pesar que los factores superan el margen sugerido (Hogarty, et al. 2005), se eliminó el factor 5 debido a que solo agrupa dos ítems (15 y 18). El modelo seleccionado se validó al obtener un valor de Kaiser-Meyer-Olkin (KMO) de 0.872 (Beavers et al. 2013) y la prueba de esfericidad de Bartlett's $=1295,5$ ( $d f=153 ; p=0,00001) p<$ 0,001 ; estos parámetros confirmaron que la prueba es idónea para realizar un AFE. El análisis factorial confirmatorio (AFC), permitió corroborar el modelo identificado en el AFE (Bollen 1989), porque, presenta un ajuste aceptable a los datos: $C F I=0,901 ; T L I=0.892$, ligeramente por debajo de $>0.09$. y RMSEA $=0,064$, se considera un modelo plausible (Kaplan, 2009; Mulaik 2009). Los índices de estimación tienen cargas mayores > 0.3 (Tabla 3) por lo que, se juzga correcto mantener en el modelo.

El cuestionario quedo conformado por 16 ítems agrupados en 4 factores subyacentes sugeridas por el modelo; El resultado dio lugar a la construcción conceptual de las 4 dimensiones: predisposición, referida a la actitud o disposición del alumno hacia el aprendizaje de las ciencias básicas, antes de iniciar las actividades académicas. Se manifiesta, por sus preferencias en la realización de actividades en comparación a otras materias de aprendizaje, está compuesta por los ítems: 2, 4 y 7 . Afecto, es un componente emocional, referido a los sentimientos que se 
desprenden de la interacción con el aprendizaje de las ciencias básicas, como el disfrute, activación, autonomía y competencia (Krapp y Lewalter (2001) y Schiefele (1991; 1999), compuesto por los ítems: 1, 3, 5, 6 y 13. Intencionalidad, es la posición deliberada hacia el estudio de las ciencias, debido a la compatibilidad con sus metas o aspiraciones. No requiere de estímulos externos para implicarse en tareas relacionadas con el objeto del estudio, Krapp y Lewalter (2001) y Schiefele (1991; 1999, está integrado por los ítems: 14, 16 y 17. Valoración, grado de importancia, por la utilidad que representa el aprendizaje de las ciencias; debido a la contribución de su desarrollo personal y la ayuda practica para la resolución de problemas (Krapp y Lewalter (2001) y Schiefele (1991; 1999), integrado por los ítems: 8,9,10,11,12.

Los resultados develan que el interés individual, es un constructo multidimensional (Schiefele, (2009); Krapp y Prenzel (2011), que integra componentes relacionados con las emociones, la predisposición, el afecto, la intencionalidad y el valor. La investigación permitió capturar más elementos para la construcción dimensional del constructo de interés individual, esto contribuye a mejorar su conceptualización ya que cobertura mayor número de elementos que robustecen su constructo y su medición. Los componentes: afecto, intencionalidad y valor, propuestos por Krapp y Lewalter (2001) y Schiefele (1991; 1999), se corroboran en la presente investigación. Sin embargo, cada uno de ellos está compuesto por nuevos enunciados (ítems) que también dieron lugar a la reconstrucción de los conceptos de cada dimensión. Por ejemplo: El afecto compuesto por 5 ítems, tiene como propósito evaluar los estados emocionales del alumno frente a las ciencias; La intencionalidad, la posición deliberada del sujeto; y el valor, compuesto por 5 ítems, evalúa el grado de importancia o significado personal que tiene el aprendizaje de las ciencias. Unido a estos componentes surge un nuevo componente que hemos denominado predisposición, integrado por 4 ítems, que mide la disposición del sujeto frente al aprendizaje de las ciencias básicas, manifiesta en la preferencia frente a otras actividades. Las investigaciones para evaluar el interés dan cuenta de una variedad de modelos: el cuestionario de interés del estudio (FSI) desarrollado por Schiefele, Krapp, Wild y Winteler (1993) integro la valencia emocionales el valor y el carácter intrínseco; su versión final está compuesto por 28 ítems. El estudio confirmo los componentes que postula la teoría relacionados con las emociones y los valores, ambos fuertemente separados y no como elementos unidimensionales. PISA 2006, desarrollo un cuestionario para medir el interés general de los estudiantes en la ciencia, el cuestionario (P21) tiene ocho ítems de opción múltiple y con escalas alto, medio, bajo y sin interés, la estrategia consiste en preguntar a los estudiantes cuánto interés tienen en aprender temas generales de ciencias: física, química, biología, astronomía, entre otros. Los elementos de interés están relacionados con el contexto y contenido; los datos del cuestionario no especifican las subcategorías cognitivas y afectivas, postuladas en la presente investigación. Otro instrumento que desarrollado por: Haeussler, 1987; Haeussler y Hoffmann, 2000 se acerca a un modelo tridimensional para evaluar el interés por la física, con la diferencia que cada una de las tres dimensiones (temas, contexto, actividades) se desgloso en subcategorías, generando un modelo complejo de ocho sub categorías de contenido, siete áreas de contexto y 4 de ocupaciones; dando lugar a 88 ítems, comparado con nuestros resultados existen diferencias sustanciales en su concepción diseño. Lamb, et al. (2012), validaron el cuestionario RASCH de la encuesta de interés de ciencia, el cuestionario contiene 18 ítems agrupados en tres dimensiones: Sentimiento, valor y la orientación intrínseca. El cuestionario contiene dos dimensiones validadas en el presente cuestionario (sentimiento y valor), así mismo, los enunciados de los ítems se basan en valoraciones que perciben los encuestados y la evaluación se realiza mediante una escala de respuesta de 4 niveles. Rotgans (2015) publico el cuestionario de interés individual (IIQ), que se puede aplicar a diferentes disciplinas, según el autor. Con una muestra de 230 estudiantes de secundaria de química, geografía e historia y 82 estudiantes de biología, valido el cuestionario mediante el análisis factorial confirmatorio. El cuestionario involucra aspectos como: el compromiso cognitivo, curiosidad, disfrute, autoeficacia, atención, 
aburrimiento y el conocimiento sobre la materia; está compuesto por 7 enunciados, con escala de 1 al 5, donde el encuestado debe responder si es cierto o no cada uno de los enunciados, es un cuestionario con distintos aspectos dimensionales, con un solo enunciado por dimensión.

El resultado ha posibilitado incorporar nuevos aspectos al constructo del interés individual, vinculados con la preferencia del alumno para realizar una determinada actividad (predisposición), también involucra el componente sentimental, debido a la interacción del alumno con el objeto de estudio (afectivo). Rescata la importancia del alumno respecto a la utilidad del contenido, la cual, provoca una jerarquía de valores (valor), capaz de direccionar las tendencias del alumno, hacia el aprendizaje de las ciencias (intencionalidad). El resultado ha permitido enriquecer el constructo, recogiendo propuestas teóricas compartidas por muchos investigadores y se han identificado más elementos que dan sostenibilidad a la complejidad del concepto (Rotgans, 2015). En este plano es importante el factor predisposición como dimensión subyacente identificada, un nuevo elemento no previsto en las diferentes modelos de concepción y evaluación del interés individual. Este factor emergente en el AFE y confirmado en el AFC, contribuye a incrementar el conocimiento y el análisis sobre la variable; teniendo en cuenta que el proceso de aprendizaje implica una constante interacción entre el sujeto y el objeto de estudio, ambos componentes tienen efectos recíprocos (Fernández, 2012), que eventualmente condicionan las expectativas y las preferencias de los alumnos, hacia el aprendizaje de las ciencias (Ausbel 2002).

Por otro lado, el instrumento aporta otros elementos significativos. Está diseñado como una herramienta suficientemente flexible para administrarse en cualquiera de las materias escolares. Sus enunciados están redactados para adaptarse en objetivos similares, reemplazando el nombre de la materia; puede aplicarse a alumnos de educación secundaria, entre 11 y 18 años. Los 16 ítems que lo componen ha demostrado que el instrumento tiene una alta capacidad para medir el constructo del interés individual. La solución del AFE explica el $83,34 \%$ de la varianza total, confirmada por el AFC donde RMSEA $=0.064$, un modelo es plausible (Kaplan, 2009; Mulaik 2009). También está demostrado que tiene una alta consistencia interna, los resultados de la prueba de alfa ordinal demuestran que los índices de alfa están por encima de 0.79 , lo cual, demuestra su alta consistencia interna.

\section{Referencias bibliográficas}

Ainley, M. (2008). Interés: un importante hilo de unión cognitiva y afectiva en la regulación del aprendizaje. Int J Psychol, 43, (4). 17-18.

Ausubel, D. P. (2002). Adquisición y retención del conocimiento. Una perspectiva cognitiva. Barcelona: Ed. Paidós.

Bartlett, M. S. (1950). Tests of significance in factor analysis. British Journal of Psychology, 3, 7785.

Bartlett, M. S. (1951). A further note on tests of significance. British Journal of Psychology, 4, 12.

Beavers, A. S., Lounsbury, J. W., Richards, J. K., Huck, S. W., Skolits, G. J., \& Esquivel, S. L. (2013). Practical Considerations for Using Exploratory Factor Analysis in Educational Research. Practical Assessment, Research \& Evaluation, 18(6). Recuperado de: http://pareonline.net/getvn.asp?v=18\&n=6.

Berlyne, D. E. (1960). Conflicto, excitación y curiosidad. Nueva York: Mc-Graw-Hill.

Blalock, H. M. (1966). Estadística Social. México: Fondo de Cultura Económica. 
Boekaerts, M., Boscolo, P. (2002). Interest and learning, learning to be interested. Learning and Instruction, 12, (1). 375-382.

Bollen KA. (1989). Structural equations with latent variables. New York: John Wiley \& Sons.

Busquets, T., Silva, M. y Larrosa, M. (2016). Reflexiones sobre el aprendizaje de las ciencias naturales. Nuevas aproximaciones y desafíos. Estudios Pedagógicos, 42, (19). 117-135. Recuperado de https://scielo.conicyt.cl/scielo.php?script=sci abstract\&pid=S0718$\underline{07052016000300010 \& \operatorname{lng}=e s \& n r m=i s o}$

De Vellis, R. F. (2012). Scale Development, Theory and Applications. Los Angeles: SAGE

Dien, J. (2010). Evaluating two-step PCA of ERP data with Geomin, Infomax, Oblimin, Promax, and Varimax rotations. Psychophysiology, 47, (1). 170-183.

Ding, C. y Hershberger, S. (2002). Assessing content validity and content equivalence using structural equation modeling. Taylor, Francis Online, 9. (2). 283-297. Recuperado de https://doi.org/10.1207/S15328007SEM0902 7

Fernández, J. (2012). La triangulación epistemológica en la interpretación del proceso de enseñanza-aprendizaje de la medicina. Educ Med Super, 26. (3), 459-466. Recuperado de http://scielo.sld.cu/scielo.php?pid=S0864$21412012000300011 \&$ script $=$ sci arttext\&tlng $=p t$

Furr, M. (2011). Scale construction and Psychometrics for social and personality Psychology. Los Ángeles: SAGE Publications.

Gorsuch, R. L. (1973). Using Bartlett's Significance Test to Determine the Number of Factors to Extract. Educational and Psychological Measurement, 33, 361-364.

Haeussler, P. (1987). Medición del interés de los estudiantes en la física: diseño y resultados de un cruce estudio seccional en la República Federal de Alemania. Revista Internacional de Ciencia Educación, 9, (1), 79-92.

Haeussler, P., Hoffmann, L. (2000). Un marco curricular para la educación física: desarrollo, comparación con los intereses de los estudiantes e impacto en el rendimiento de los estudiantes y su autoestima concepto. Science Education, 84, (1), 689-705.

Harman, HH. (1962). Análisis factorial moderno. Prensa de la Universidad de Chicago, Chicago.

Hazelkorn, E., Ryan, C., Beernaert, Y., Constantinou, C.P., Deca, L., Grangeat, M., Karikorpi, M., Lazoudis, A., Pintó Casulleras, R., y Welzel-Breuer, M. (2015). Science education for responsible citizenship. Report to the European Commission of the expert group on science educatión. Recuperado de: http://ec.europa.eu/research/swafs/pdf/pub_science_education/KI-NA-26 -893-ENN.pdf $(25 / 11 / 2016)$.

Hendrickson, A. E., White, P. O. (1964). Promax: a quick method for rotation to oblique simple structure. British Journal of Statistical Psychology, 17, 65-70.

Hidi, S. y Baird, W. (1986). Intereses: una variable descuidada en el procesamiento del discurso. Cogn Sci, 10, (2). 179-194.

Hidi, S. y Renninger, K. (2006). El modelo de cuatro fases de desarrollo de intereses. Educ Psychol, 41, (2). 111-127. 
Hogarty, K., Hines, C., Kromrey, J., Ferron, J. y Mumford, K. (2005). The quality of factor solutions in exploratory factor analysis: The influence of sample size, communality, and overdetermination. Educational and Psychological Measurement, 65, 202-226.

Kaiser, H. F. (1970). A second generation Little Jiffy. Psychometrika, 35, 401-415.

Kaplan, D. (2009). Structural equation modeling: foundations and extensions. Segunda edición. Thousand Oaks, CA: Sage. Recuperado de Doi:10.4135/9781452226576

Kelly, W. (1982). Psicología de la educación. Recuperado de https://books.google.com.pe/books?id=ECaz9yx8MScC\&printsec=frontcover\&hl=es\&so urce $=$ gbs ge summary $r \& c a d=0 \# v=o n e p a g e \& q \& f=f a l s e$

Krapp, A. y Lewalter, D. (2001). Development of interest and interest-based motivational orientations: a longitudinal study of vocational school and work settings. En S. Volet y S. Järvelä (Eds). Motivation in learning contexts: theoretical advances and methodological implications. Ámsterdam, The Netherlands: Pergamon

Krapp, A. y Prenzel, M. (2011). Research on interest in science: Theories, methods and findings. International Journal of Science Education, 33, (1). 27-50.

Krapp, A., Hidi, S., \& Renninger, K. A. (1992). Interest, learning, and development. In K. A. Renninger, S. Hidi, \& A. Krapp (Eds.), The role of interest in learning and development (p. 3-25). Lawrence Erlbaum Associates, Inc.

Lamb, R., Annetta, L., Meldrum, J. y Vallett, D. (2012). Measuring science interest: Rasch validation of the science interest survey. International Journal of Science and Mathematics Education, 10 (3). 643-668. Recuperado de DOI: 10.1007/s10763-011-9314$z$

Lorenzo-Seva, U. y Ferrando, P. (2006). FACTOR: a computer program to fit the exploratory Factor Analysis model. Behavioral Research Methods, 38, (1). 88-91. Recuperado de DOI: $10.3758 /$ bf03192753

Lorenzo-Seva, U., Ten Berge, JMF (2006). El coeficiente de congruencia de Tucker como un índice significativo de similitud factorial. Metodología 2, 57-64.

Martínez Arias, R. (1995). Psicometría. Madrid: Síntesis Psicológica.

Mulaik, S. A. (2009). Linear causal modeling with structural equations. New York: CRC Press Taylor \& Francis Group. Recuperado de Doi: 10.1201/9781439800393

Muthén, B. (2004). Latent variable analysis: Growth mixture modeling and related techniques for longitudinal data. In D. Kaplan (ed.), Handbook of quantitative methodology for the social sciences. Newbury Park, CA: Sage Publications.

Muthén, L.K. y Muthén, B.O. (1998-2010). Mplus User's Guide. Los Angeles, CA: Muthén \& Muthén.

O'Keefe, P., Linnenbrink, L. (2014). El papel del interés en optimizar el rendimiento y la autorregulación. Revista de psicología social experimental, 53, (2). 70-78. Recuperado de https://doi.org/10.1016/i.jesp.2014.02.004

Pérez-Gil, J., Chacón, S. y Moreno, R. (200). Validez de constructo: el uso de análisis factorial exploratorio-confirmatorio para obtener evidencias de validez. Psicothema, 12, (2), 442446. Recuperado de http://www.redalyc.org/articulo.oa?id=72797102

Rennie, L. y Punch, K. (1991). La relación entre afecto y logro en Ciencias. Revista de Investigación en Enseñanza de las Ciencias, 28 (2), 193-209. 
Renninger, K y Hidi, S. (2011). Revisando la conceptualización, medición y generación de interés. Educ Psychol, 46, (3). 168-184. Recuperado de https://doi.org/10.1080/00461520.2011.587723

Rocard, M., Csermely, P., Jorde, D., Lenzen, D., Walberg-Henriksson, H., y Hemmo, V. (2007). Science education now: A renewed pedagogy for the future of Europe. Report to the European Commission of the expert group on science education. Recuperado de http://ec.europa.eu/research/science-society/document_library/pdf_06/report-rocardon-science-education_en.pdf (25/11/2016).

Rotans, J. (2015). Estudio de validación de una medida de interés general de la materia: el cuestionario de interés individual (IIQ). Revista Educación de profesiones sanitarias, 1, (1), 67-75. Recuperado de https://doi.org/10.1016/j.hpe.2015.11.009

Rotgans, J. y Schmidt, H. (2011). El papel de los maestros para facilitar el interés situacional en un aula de aprendizaje activo, Teach Teach Educ, 27, (1). 37-42.

Rotgans, J. y Schmidt, H. (2014). Interés situacional y aprendizaje: la sed de conocimiento Aprender Instr, 32, (2). 37-50.

Schiefele, U. (1991). Interest, learning and motivation. Educational Psychologist, 26, (3-4), 299232.

Schiefele, U. (1992). Topic interest and levels of text comprehension. Educational Psychologist. 8(2), 141-160. https://www.sciencedirect.com/science/article/pii/S1041608096900308

Schiefele, U. (1999). Topic interest and levels of text comprehension. Educational Psychologist. 8(2), 141-160. Recuperado de https://www.sciencedirect.com/science/article/pii/S1041608096900308

Schiefele, U. (2009). Interés situacional e individual. En KR Wentzel y A. Wigfield (Eds.), Manual de motivación en la escuela. Mahwah, Nueva Jersey: Erlbaum.

Schiefele, U., Krapp, A. y Schreyer, I. (1993). Meta análisis de la relación entre interés y rendimiento escolar. Revista de psicología del desarrollo y educativo, 25, 82). 120-148.

Schiefele, U., Krapp, A., Wild, K. -P., y Winteler, A. (1993). El cuestionario sobre Studieninteresse (FSI). (El cuestionario sobre el interés del estudio). Diagnostica, 39, (1). 335-351.

Silvia, PJ (2005). ¿Qué es interesante? Explorando la estructura de evaluación de interés. Emoción, 5, (1), 89.

Steinkamp, MW, y Maehr, ML (1983). Afecto, habilidad y logro científico: A síntesis cuantitativa de la investigación correlacional. Revisión de Investigación Educativa, 53, (2). 369-396.

Tabachnick, B. G. y Fidell, L. S. (2007). Using multivariate statistics. Boston: Pearson/Allyn \& Bacon.

Ten Berge, JMF., Kiers, HAL (1991). Un acercamiento numérico al rango mínimo exacto y aproximado de una matriz de covarianza. Psychometrika 56, 309-315.

Thompson, B. (2008). Exploratory and Confirmatory Factor Analysis, Understanding concepts and applications. Washington DC: American Psychological Association.

Velicer, W. y Fava, J. (1998). Effects of variable and subject sampling on factor pattern recovery. Psychological Methods, 3, (2). 231-251. Recuperado de doi:10.1037/1082-989x.3.2.231 
Velicer, W. y Fava, J. (1998). Effects of variable and subject sampling on factor pattern recovery. Psychological Methods, 3, (2), 231-251. Recuperado de doi:10.1037/1082-989x.3.2.231

Winteler, A. y Sierwald, W. (1987). Desarrollo y revisión de un cuestionario sobre Interés de estudio (FSI). educación superior, 5, (2). 223-242.

Winteler, A., Sierwald, W. y Schiefele, U. (1988). Interés, desempeño y conocimiento. Pedagogía empírica, 2, (1). 227-250.

Yanai, H. y Ichikawa, M. (2007). Factor Analysis. En C. R. Rao \& S. Sinharay (editors). Handbook of Statistics 26. Psychometric. Holanda norte: Elsevier. 